\title{
Effects of cisplatin on photosensitizer-mediated photodynamic therapy in breast tumor-bearing nude mice
}

\author{
Tae-Gyu Ahn, Ji Min Jung, Eun-Jeong Lee, Ji Hyun Choi \\ Department of Obstetrics and Gynecology, Chosun University College of Medicine, Gwangju, Korea
}

Objective

This study aimed to evaluate the potential effects of cisplatin on photodynamic therapy (PDT) in breast cancer using a breast tumor-bearing mouse model.

Methods

In this study, breast tumor (experimental mammary tumour-6 cell)-bearing nude mice were used as experimental animals. Photolon ${ }^{\circledR}$ (photosensitizer, $2.5 \mathrm{mg} / \mathrm{kg}$ body weight [BW]) was injected intraperitoneally; after 2 hours, the tumors were irradiated $\left(660 \mathrm{~nm}, 80 \mathrm{~J} / \mathrm{cm}^{2}\right)$ using a diode laser tool. Cisplatin $(3 \mathrm{mg} / \mathrm{kg} \mathrm{BW})$ was injected intraperitoneally 1 hour before the Photolon ${ }^{\circledR}$ injection.

\section{Results}

Tumor volume increased over time in the control group and was not different from that in the cisplatin group. In the PDT group, the tumor volume increased on day 3, but not on day 7. In the cisplatin+PDT group, tumor volume increased on day 3 but decreased on day 7. There was no significant difference in the levels of thiobarbituric acid reactive substance (TBARS) in tumor tissues between the control and cisplatin groups. The levels of TBARS in the cisplatin+PDT group were higher (47\%) than those in the PDT group. Analysis of tumor tissue transcriptomes showed that the expression of genes related to the inflammatory response including $C L$ and $X C L$ genes increased, while that of Fn1 decreased in the cisplatin+PDT group compared with the PDT group.

\section{Conclusion}

These results suggest that cisplatin enhances the therapeutic effect of PDT in a breast tumor-bearing mouse model. However, further clinical studies involving patients with breast cancer is needed.

Keywords: Photodynamic therapy; Cisplatin; Breast cancer; Nude mice

\section{Introduction}

The prevalence of breast cancer is high, with a mortality rate of $23 \%$ among female cancer patients and $14 \%$ among all cancer patients worldwide [1]. Current therapies for patients with breast cancer include surgery, chemotherapy, and radiation therapy; surgery is considered the definitive treatment. However, surgery is not recommended to all breast cancer patients, and traditional chemotherapies have multiple side effects, including the emergence of resistant cells and toxic side effects. Therefore, novel therapies and treatments are being investigated $[1,2]$.

Photodynamic therapy (PDT) is a minimally invasive therapy that involves irradiation of targeted cancerous lesions [3]. The 3 main factors involved in PDT are a photosensitizer, light, and oxygen irradiation onto the photosensitizer results in excitation, which then produces oxygen free radicals that damage the targeted cancer cells [4]. Known photosensitizers

Received: 2018.11.07. Revised: 2018.12.26. Accepted: 2018.12.27. Corresponding author: Ji Hyun Choi

Department of Obstetrics and Gynecology, Chosun University School of Medicine, 309 Pilmun-daero, Dong-gu, Gwangju 61452, Korea E-mail: themoon6pence@hanmail.net https://orcid.org/0000-0001-7538-6129

Articles published in Obstet Gynecol Sci are open-access, distributed under the terms of the Creative Commons Attribution Non-Commercial License (http://creativecommons. org/licenses/by-nc/3.0/) which permits unrestricted non-commercial use, distribution, and reproduction in any medium, provided the original work is properly cited.

Copyright $\odot 2019$ Korean Society of Obstetrics and Gynecology 


\section{Obstetrics \& Gynecology Science}

Tae-Gyu Ahn, et al. Cisplatin effect on photodynamic therapy

used in PDT include porphyrin-family material, chlorine-family material, and phthalocyanines [5]. Photofrin ${ }^{\circledR}$, a porphyrin derivative, was first used in Canada (1993) for the treatment of bladder cancer and has since been used for the treatment of different cancers, including esophageal and lung cancer, in the US, Japan, and Europe [4,5]. Because Photofrin ${ }^{\circledR}$ is not degraded and remains intact inside the cell for several weeks, patients who receive PDT using Photofrin ${ }^{\circledast}$ are advised to cover their skin, apply sunblock, and wear sunglasses in order to avoid exposure to sunlight [5]. Photolon ${ }^{\circledR}$ is a watersoluble material synthesized by mixing the chlorine derivative e6 with polyvinylpyrrolidone [6]. Photolon ${ }^{\circledR}$ accumulates within the cancer cell approximately 1 hour after intravenous injection and most of the Photolon ${ }^{\circledR}$ in the body is excreted after 12 hours. This allows patients to resume normal activity within a relatively short period after PDT [7]. However, low levels of photoreaction have been reported following the injection of photosensitizer due to low solubility and potential interaction with other biomolecules. Consequently, different attempts have been made (i.e., development of novel drugs, combination therapy with chemotherapeutic agents) to improve treatment effectiveness $[3,4,8]$.

Lasers are often used as a light energy source to activate the photosensitizer. The laser used in PDT has a low power output, and a diode laser is commonly used due to its low cost and convenient management [9]. The wavelength of the laser used in PDT differs depending on the photosensitizer; for porphyrin- or chlorine-family material, wavelengths of 600-700 nm (red light) are used, since they are activated within this range of wavelengths [10].

Cisplatin, also known as cis-platinum or cis-diamminedichloroplatinum (II), is a broad-spectrum anticancer drug; it is a chemotherapeutic agent used for the treatment of different types of cancers [11]. Cisplatin is effective against different cancer types, including carcinoma, sarcoma, and embryoma. It is widely used for the treatment of various cancer types including cancers of the head and neck, breast, lungs, colon, bladder, and genital organs [12]. Nevertheless, the emergence of cells that are resistant to cisplatin [13] and the side effects associated with the drug (i.e., renal damage, gastric disorders, and auditory nerve damage) have limited its use in humans [14]. Due to its side effects and associated resistance, cisplatin-based chemotherapy is usually used in combination with different chemotherapeutic agents or other treatment methods, including PDT [15]. Ali et al. [16] utilized a myosarcoma cell line and reported that a combination of cisplatin and PDT resulted in increased anticancer activity, while Wei et al. [17] demonstrated that a combination of cisplatin and 5 -aminolevulinic acid in a cervical cancer cell line (HeLa) increased the effectiveness of PDT. This study aimed to assess the effect of cisplatin on PDT in breast cancer using nude mice injected with a breast cancer cell line line (experimental mammary tumour-6 [EMT6] cells) to create a breast tumor-bearing mouse model. Mice were injected with Photolon ${ }^{\circledR}$ and cisplatin, and then subjected to PDT. Different treatment outcomes were assessed, including tumor volume, lipid peroxidation in tumor tissue, and changes in the expression of genes associated with the inflammatory response.

\section{Materials and methods}

\section{Experimental materials}

Cisplatin was obtained from Sigma-Aldrich Co. (St. Louis, MO, USA), while fetal bovine serum (FBS), streptomycin, penicillin, and Dulbeco's Modified Eagle's Media (DMEM) were obtained from Gibco BRL (New York, NY, USA). The thiobarbituric acid reactive substance (TBARS) Assay Kit was purchased from KOMA Biotech Inc. (Seoul, Korea), and Photolon ${ }^{\circledR}$ was purchased from Belmedpreparaty (Minsk, Belarus). The EMT6 cell line (Mus musculus breast carcinoma cells) was purchased from American Type Culture Collection.

\section{Establishment of a breast cancer-bearing mouse model}

The experimental animals used in this study were generated from the nude mouse strain KSN/Slc at 6 weeks of age, purchased from the Central Lab Animal Inc. (Seoul, Korea). Mice were reared under a 12-hour day-night cycle, temperature of $20 \pm 2^{\circ} \mathrm{C}$, and relative humidity of $60 \pm 5 \%$. Animal experiments were performed according to the animal research ethics protocol, with approval from the Animal Research Ethics Board of Chosun University (approval number: CIACUC 2015-A0034). The experimental mice underwent a 1-week adaptation period to the new environment prior to the induction of breast cancer. Breast cancer cells (EMT6) were cultured in DMEM containing 10\% FBS, streptomycin (100 $\mathrm{U} / \mathrm{mL})$, and penicillin $(100 \mathrm{U} / \mathrm{mL})$, in a $\mathrm{CO}_{2}$ incubator at $37^{\circ} \mathrm{C}$. Cultured murine breast cancer cells were harvested and resuspended in PBS, and $0.2 \mathrm{~mL}\left(2 \times 10^{5}\right.$ cells $)$ of this mixture 


\section{Obstetrics \& Gynecology Science}

Vol. 62, No. 2, 2019

was injected subcutaneously in the back of a nude mouse. After injection, the mice were monitored for potential tumor development, and the size of the tumor was measured using a digital caliper (Mitutoyo Korea, Busan, Korea). Mice that developed up to 9 -mm tumors 10 days after the injection were used in the experiment.

\section{Photodynamic therapy}

Mice with induced tumors (size: $\geq 9 \mathrm{~mm}$ ) were divided into 4 groups, with 10 mice in each group: control group, cisplatin group, PDT group, and combination (cisplatin+PDT) group. Cisplatin was diluted in normal saline solution and injected into the abdominal cavity of each mouse $(3 \mathrm{mg} / \mathrm{kg}$ mouse BW) 1 hour prior to Photolon ${ }^{\circledR}$ injection. Similarly, Photolon ${ }^{\circledR}$ was diluted in normal saline solution and injected into the abdominal cavity of each mouse (2.5 mg/kg mouse BW). Finally, PDT was performed 2 hours after Photolon ${ }^{\circledR}$ injection by emitting a non-thermal laser light (Ceralas ${ }^{\mathrm{T}}{ }^{\mathrm{M}}$ Diode Laser 632 System; BioLitec, Germany, $660 \mathrm{~nm}, 80 \mathrm{~J} / \mathrm{cm}^{2}$ ) onto the cancerous lesion.

Tumor mass was assessed in 5 mice from each group before and after PDT (in different time points) until day 7, and images were obtained. Tumor size was measured using a digital caliper, and the volume was calculated using the following equation: tumor volume $\left(\mathrm{mm}^{3}\right)=\left(\right.$ width ${ }^{2} \times$ length $) / 2$

\section{Measurement of thiobarbituric acid reactive substance within the tumor tissue}

Twenty-four hours after PDT, 5 mice from each group were sacrificed and the tumor tissues were resected. Part of the resected tumor was used as to determine the amount of TBARS, which is a product of lipid peroxidation.

To prepare samples for TBARS measurement, $0.3 \mathrm{~g}$ of tissue was resected and mixed with $3 \mathrm{~mL}$ of $0.1 \mathrm{M}$ phosphate buffer ( $\mathrm{pH}$ 7.0) prior to homogenization with a polytrone homogenizer. The amount of TBARS was measured using the OxiSelect ${ }^{\mathrm{TM}}$ TBARS Assay Kit (KOMA Biotech Inc.) according to the manufacturer's protocol.

\section{Transcriptome profiling of the tumor tissues}

Part of the tumor tissue was resected, and total RNA was extracted using TRIzol ${ }^{\otimes}$ RNA Isolation Reagent (Life Technologies, Carlsbad, CA, USA). Using the RNA extracted from the tumor tissue, an mRNA sequencing library was created using the TruSeq stranded mRNA sample preparation kit (Illumina,
San Diego, CA, USA), according to the manufacturer's protocol. The library was subsequently analyzed using an Agilent DNA High Sensitivity Kit (Agilent, Santa Clara, CA, USA) and BioAnalyzer 2100. Ultimately, the library was sequenced on the Illumina HiSeq 2500 platform for subsequent RNA sequencing analysis. To generate a cDNA library from the tumor tissues, clusters of cDNA libraries were created using TruSeq flow cell, and sequences were analyzed using the TruSeq 200 Cycle SBS kit (Illumina), which produced 100-bp end reads. The sequencing results of CDNA libraries generated by Illumina HiSeq 2500 were comparatively analyzed with the information stored in FASTQ format. Gene sets were analyzed using the Functional Annotation Tool from DAVID Bioinformatics Resources 6.7, NIH (http://david.abcc.ncifcrf.gov) [18]. Differences in gene expression among the experimental groups were assessed using the fold-change false discovery rate, calculated using reads per kilobase per million mapped reads, with $P<0.05$ denoting statistically significance.

\section{Statistical analyses}

All measurements, except for RNA-seq results, were represented by mean \pm standard deviation, and the experimental outcomes were analyzed with analysis of variance using Statistical Package for the Social Science Ver. 12.0 (SPSS Inc., (hicago, IL, USA). Significance among samples was assessed using Duncan's multiple range test, at $P<0.05$.

\section{Results}

\section{Visual evaluation of tumors in a mouse model of breast cancer after photodynamic therapy}

To evaluate the effects of PDT, tumors were imaged on day 0 (immediately before PDT), day 3 after PDT, and day 7 after PDT (Fig. 1).

The size of tumors in the control group was increased on days 3 and 7 compared with day 0 . On day 7 , the tumor was enlarged and the skin surface in the center of tumor was expanded, causing the subcutaneous tissue and blood vessels to become visible. No hemorrhage or necrosis was observed. No visible difference was observed between the cisplatin and control groups.

The middle section of tumors in the PDT group exhibited a change in color (to black) on days 3 and 7. No change in color was observed in the surrounding area. In the combina- 


\section{Obstetrics \& Gynecology Science}

Tae-Gyu Ahn, et al. Cisplatin effect on photodynamic therapy

tion group, most tumor tissues exhibited a change in color (to black), and the middle section of the tumor was slightly sunken. On day 7, the middle section of the tumor peeled off and the subcutaneous layer was visible.

\section{Changes in tumor volume in a mouse model of breast cancer after photodynamic therapy}

To observe the effect of PDT on tumor growth, the tumor volume was measured on days 0, 3, and 7 after PDT (Fig. 2). Compared with the tumor volume on day 0 , those on days 3 and 7 were enlarged by $21 \%$ and $135 \%$ respectively, demonstrating that the tumor grows over time.

Tumor volumes in the cisplatin group were increased by $19 \%$ and $110 \%$ on days 3 and 7 compared with that on day 0 , with a slight difference in the rate of tumor growth compared with that in the control group. In the PDT group, the tumor volumes on days 0,3 , and 7 were $395 \pm 101$, $697 \pm 143$, and $691 \pm 154 \mathrm{~mm}^{3}$, respectively, indicating an increase of $67 \%$ and $74 \%$ on days 3 and 7 , respectively. De-

Cisplatin
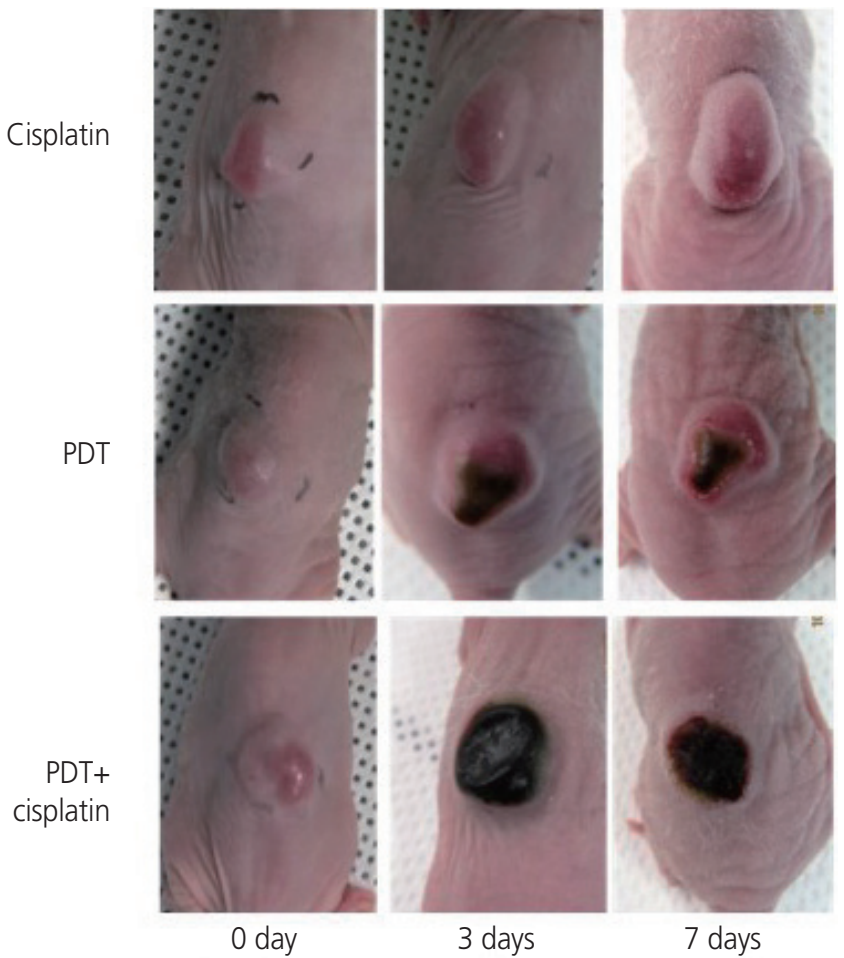

Fig. 1. Photograph of breast tumor-bearing mice after PDT. Diode lazer light $\left(660 \mathrm{~nm}, 80 \mathrm{~J} / \mathrm{cm}^{2}\right)$ was used to irradiate the tumor mass of mice 2 hours after intra-peritoneal injection of Photolon ${ }^{\circledR}$ (2.5 mg/kg). Cisplatin (3 mg/kg) was injected intraperitoneally 1 hour before Photolon ${ }^{\circledR}$ treatment. PDT, photodynamic therapy. spite the greater rate of tumor growth on day 3 compared with the control group, the rate significantly reduced on day 7 and the rate of tumor growth was lower in the PDT group. Tumor volumes in the combination (cisplatin+PDT) group were $401 \pm 107,711 \pm 141$, and $612 \pm 131 \mathrm{~mm}^{3}$ on days 0,3 , and 7 , respectively, increasing by $77 \%$ and $52 \%$ on days 3 and 7, respectively. In the PDT group, although tumor growth was evident on day 3, tumor volume on day 7 was lower than that on day 3 (Table 1).

\section{Changes in lipid peroxidation products in a mouse model of breast cancer after photodynamic therapy}

To understand the effect of PDT on the amount of lipid peroxidation, mice were sacrificed 1 day after PDT and the amount of TBARS from the tumor tissue was measured (Fig. 2).

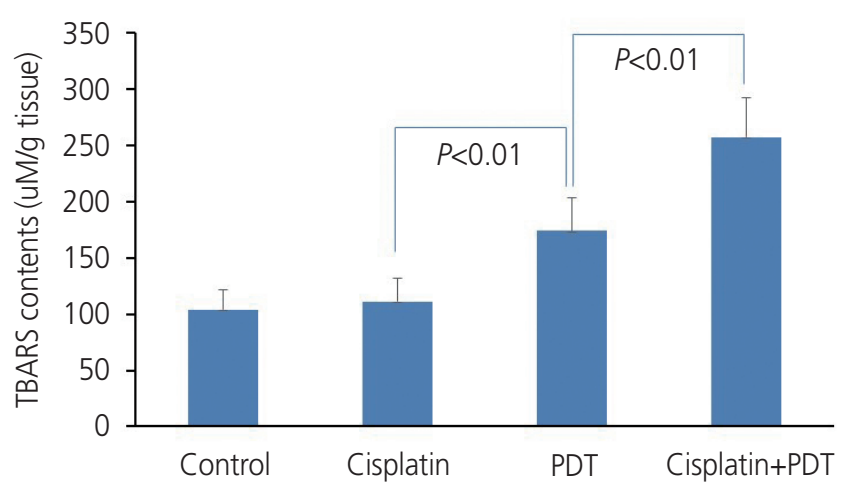

Fig. 2. TBARS in tumor tissue of tumor-bearing mice after treatment with PDT. TBARS contents in tumor tissue of tumor bearingmice were measured 24 hours after PDT using OxiSelect ${ }^{\text {TM }}$ TBARS assay kit. Data were analyzed using Duncan's multiple range test. TBARS, thiobarbituric acid reactive substance; PDT, photodynamic therapy.

Table 1. Effects of photodynamic therapy and cisplatin on tumor growth in breast tumor-bearing mice

\begin{tabular}{lccc}
\hline \multirow{2}{*}{ Groups } & \multicolumn{3}{c}{ Tumor volume $\left(\mathbf{m m}^{\mathbf{3}}\right)$} \\
\cline { 2 - 4 } & $\mathbf{0}$ day & $\mathbf{3}$ days & $\mathbf{7}$ days \\
\hline Control & $381 \pm 92$ & $462 \pm 112$ & $896 \pm 209$ \\
Cisplatin & $411 \pm 112$ & $491 \pm 119$ & $867 \pm 173$ \\
PDT & $395 \pm 101$ & $697 \pm 143$ & $691 \pm 154$ \\
Cisplatin+PDT & $401 \pm 107$ & $711 \pm 141$ & $612 \pm 131$
\end{tabular}

Tumors size was measured by digital caliper and tumor volume was calculated using the following formula: tumor volume $\left(\mathrm{mm}^{3}\right)=\left[(\text { width })^{2}\right.$ xlength]/2.

Values are the mean \pm standard deviation $(n=5)$.

PDT, photodynamic therapy. 


\title{
Obstetrics \& Gynecology Science
}

\author{
Vol. 62, No. 2, 2019
}

The amount of TBARS in the tumor tissue was $104 \pm 18 \mathrm{uM} / \mathrm{g}$ tissue in the control group, with similar levels observed in the cisplatin group. The amount of TBARS in the PDT group was increased by up to $70 \%$ compared with the control group. More strikingly, the amount of TBARS in the combination group was even greater $(257 \pm 41 \mathrm{uM} / \mathrm{g}$ tissue, up to $47 \%$ increase compared with the PDT group), demonstrating increased levels of oxidative damage.

\section{Comparison of inflammatory response-related gene expression in the photodynamic therapy vs. combination (cisplatin+photodynamic therapy) group}

The expression of genes related to inflammation in tumor tissues from the PDT and combination groups was analyzed and compared via mRNA-sequencing. The genes that exhibited $\log 2$ (fold change [FC]) values $\geq 1$ or $\leq-1$ and $P<0.05$ are listed in Table 2.

Compared with the PDT group, the combination group presented increased expression of the following inflammatory response-related genes in tumor tissue: CL2, CL7, and CL12. The log2 (FC) values of those genes were 1.49, 1.61, and 1.50 respectively. Similarly, the expression of $X C L 1$, $X C L 2, X C L 5$, and $X C L 10$ were increased in tumor tissue from the combination group, with $\log 2(\mathrm{FC})$ values of $3.22,4.51$, 3.63 , and 1.91, respectively. The log2 (FC) values of $I L-1 \beta$ and $I L-6$ genes were 1.29 and 1.55 , which demonstrated the

Table 2. Selective transcripts of inflammation-related genes in tumor tissue from cisplatin+photodynamic therapy mice vs. photodynamic therapy mice

\begin{tabular}{lrccc}
\hline Genes & PDT & Cisplatin+PDT & log2 (FC) & P-value \\
\hline CL2 & 94.40 & 265.53 & 1.49 & 0.00005 \\
CL7 & 13.73 & 41.83 & 1.61 & 0.00005 \\
CL12 & 18.14 & 51.15 & 1.50 & 0.00015 \\
XCL1 & 2.27 & 21.17 & 3.22 & 0.00005 \\
XCL2 & 0.62 & 14.15 & 4.51 & 0.00015 \\
XCL5 & 5.46 & 67.43 & 3.63 & 0.00005 \\
XCL10 & 5.15 & 19.41 & 1.91 & 0.00005 \\
IL-1 3 & 23.55 & 57.57 & 1.29 & 0.00005 \\
IL-6 & 17.50 & 51.13 & 1.55 & 0.00005 \\
Fn1 & 384.49 & 144.22 & -1.41 & 0.00005 \\
\hline
\end{tabular}

Transcripts were examined by RNA-seq in tumor tissue of tumorbearing mice 1 day after PDT.

PDT, photodynamic therapy; FC, fold change. increased expression of these genes in tumor tissue obtained from mice in the combination group. However, expression of the $F n 1$ gene was decreased in the combination group with a $\log 2(\mathrm{FC})$ value of -1.41 .

\section{Discussion}

In the present study, experiments using a breast cancer mouse model were performed to evaluate the effects of cisplatin combined with PDT using Photolon ${ }^{\circledast}$. In the combination group, the area of blackening on the surface of the tumor was larger, and the tumor was smaller compared with the PDT group. PDT induces localized damage to irradiated lesions, and damage to the cancer cells and vascular system within the tumor tissue can induce necrosis of the tumor tissue. Darkening of the skin that covers the tumor tissue indicates necrosis; therefore, the observed color change (to black) of the skin that covers the cancerous lesion after PDT may indicate that the tumor tissue had undergone necrosis. Following injection, photosensitizers accumulate within lysosomes or mitochondria, and irradiation with a laser causes lysosomal hydrolase to leak into the cytoplasm, resulting in cell necrosis [19]. Furthermore, PDT causes damage to vascular endothelial cells, resulting in thrombus formation and vessel blockade, and thus inducing secondary necrosis of the tumor [20].

The change in skin color in the PDT group was only observed in the middle part of the tumor tissue; this finding indicates that tissue necrosis only occurred in a specific region of the tumor. Conversely, the combination group exhibited a change in the color in the skin across the entire tumor surface, suggesting that tissue necrosis occurred throughout the tumor. These experimental findings suggest that cisplatin provides additive antitumor effects to PDT using Photolon ${ }^{\circledR}$. Moreover, since the cisplatin group exhibited no tumor growth or changes in skin color, the amount of cisplatin injected in this study (3 mg/kg mouse BW) had no or minimal effects on the growth or necrosis of cancer cells.

Cancer cell proliferation increases tumor volume. In this study, tumor volume in the control group increased by $21 \%$ and $135 \%$ on days 3 and 7 after PDT, respectively, demonstrating that the tumor is continuously growing. Mice in the cisplatin group had a similar rate of tumor growth compared with those in the normal group; therefore, cisplatin injection 


\section{Obstetrics \& Gynecology Science}

Tae-Gyu Ahn, et al. Cisplatin effect on photodynamic therapy

(3 mg/kg mouse BW) appeared to have no effect on the suppression of tumor growth. Tumor volume in the PDT group, compared with that before treatment (day 0), increased by $67 \%$ and $74 \%$ on days 3 and 7 , respectively. Additionally, the rate of tumor growth on day 3 was greater in the PDT group than in the control group. One explanation for this observation is the inflammatory response following photodynamic reaction. Nonetheless, by day 7, the rate of tumor growth was lower in the PDT group compared with the control group. Tumor volume in the combination (cisplatin+PDT) group, compared with that before treatment (day 0), was increased by $77 \%$ and $52 \%$ on days 3 and 7 , respectively. On day 3, the rate of tumor growth in the combination group was greater than that in the PDT group, which may have been caused by the greater inflammatory response following the photodynamic reaction. On day 7, both groups exhibited a decrease in tumor volume, further indicating that cisplatin injection induces an additive effect and maximizes the effectiveness of PDT using Photolon ${ }^{\circledR}$.

Following injection of cisplatin into the abdominal cavity of mice, the concentration of cisplatin within the tissue reaches maximal levels within 15 minutes and lasts for $0.5-4$ hours [21]. In this study, there was a 3-hour interval between cisplatin injection and laser irradiation, and the latter likely occurred when the concentration of cisplatin in the tissue was maximal. The lethal dose of cisplatin in mouse is $\geq 20 \mathrm{mg} / \mathrm{kg}$ [21]; however, the concentration of cisplatin used in this study was $3 \mathrm{mg} / \mathrm{kg}$, which is less than that typically used (5 $\mathrm{mg} / \mathrm{kg}$ ). The tumor size in the combination group was lesser than that in the PDT group. This change was probably a result of the interaction between cisplatin and activated Photo$\operatorname{lon}^{\circledast}$, which induced an additive effect on tumor suppression. Ali et al. [16] treated a myosarcoma cell line with PDT, using a phthalocyanine-family photosensitizer, aluminum phthalocyanine tetrasulfonate chloride and demonstrated that injection of low-dose cisplatin resulted in increased anticancer activity. In addition, Ge et al. [8] suggested that cisplatin increased anticancer activity in a colorectal cancer cell treated with PDT using a photosensitizer called Photogem ${ }^{\circledR}$. In this study, injection of cisplatin increased the effectiveness of PDT using Photolon ${ }^{\circledR}$ in a breast cancer mouse model, producing a similar outcome as those reported in previous studies.

The therapeutic effect of PDT depends on the photosensitizer producing free radicals through a photodynamic reaction. When the photosensitizer is irradiated, the photody- namic reaction occurs and produces reactive oxygen species (ROS) [22]. As cisplatin increases ROS synthesis [23], cisplatin combined with PDT may promote ROS synthesis. In turn, ROS induces lipid peroxidation. In the present study, the levels of lipid peroxidation products in the tumor tissue was measured by assessing the amount of TBARS. The amount of TBARS in the tumors of PDT group was increased by up to $70 \%$ compared with that in the control group, indicating an increased amount of lipid peroxidation product from PDT. Furthermore, the amount of TBARS in the tumors of the combination (cisplatin+PDT) group was greater than that in the PDT group (up to $47 \%$ ), further supporting the hypothesis that a combination of cisplatin and PDT increased the oxidative damage of cancer cells. Localized inflammation following PDT is a key process of this anticancer therapy; this response involves multiple different factors, including vasoactive substances, the complement system, acute-phase proteins, proteinases, peroxidases, ROS, white blood cell chemoattractants, cytokines, growth factors, and other immune response mediators [24].

To examine the effect of cisplatin on the expression of inflammatory response-related genes within tumor tissues subject to PDT, the tumor tissue transcriptome was analyzed with mRNA-sequencing on day 1 after PDT and the outcomes from the PDT group and combination (cisplatin+PDT) group were compared. Inflammatory response-related genes with a $\log 2(F C)$ value $\geq 1$ and $P<0.05$ included $C L 2, C L 7$, CL12, XCL1, XCL2, XCL5, XCLI0, IL-1 $\beta$, and IL-6. Conversely, Fn1 was the only gene with a $\log 2(F C) \leq-1$ and $P<0.05$.

CL2 and CL7 are secreted from macrophages and exhibit chemotaxis towards monocytes $[25,26]$. CL12 is secreted from macrophages but exhibits chemotaxis towards monocytes, eosinophils, and lymphocytes [27]. XCL2 is secreted from monocytes and macrophages, exhibiting chemotaxis towards polymorphonuclear leukocyte [28], and XCL5 is secreted from endothelial cell or neutrophils during the acute inflammatory response to activate neutrophils [29]. XCL10 is secreted from monocytes or endothelial cells, and exhibits chemotaxis towards monocytes and macrophages [30]. IL-1 $\beta$ and $I L-6$ are secreted from activated macrophages to induce an inflammatory response [31].

Fn1 is involved in intracellular connections within the tissue, and promotes repair of tissue damage [32]. Compared with the PDT group, the combination group exhibited increased expression of genes involved in chemotaxis (i.e., CL2, CL7, 


\section{Obstetrics \& Gynecology Science}

Vol. 62, No. 2, 2019

CL12, XCL1, XCL2, XCL5, and XCLI0) to recruit and activate cells involved in the inflammatory response (i.e., monocytes, eosinophils, neutrophils, and macrophages). In addition, the levels of $I L-1 \beta$ and $I L-6$ were increased, which promote inflammation. Together, the increased expression of these genes is thought to drive further inflammation in the tumor tissue. Conversely, the expression of Fn1, which suppresses the immune response and promotes wound healing, was decreased. The increased inflammatory response in the combination group, compared with the PDT group, is thought to result in an increased anticancer effect.

Overall, these experiments demonstrated that, in a breast cancer mouse model treated using PDT with Photolon ${ }^{\circledR}$, a combination of PDT and low-dose cisplatin resulted in an increase cancer cell necrosis, oxidative damage in the tumor tissue, and inflammation within the tumor tissues. Therefore, we suggest that cisplatin treatment combined with PDT may bring additive therapeutic effects for the treatment of cancerous lesions, although additional future studies are needed to confirm these findings.

\section{Acknowledgements}

This study was supported by research fund from Chosun University, 2018.

\section{Conflict of interest}

No potential conflict of interest relevant to this article was reported.

\section{Ethical approval}

Animal experiments were performed according to the animal research ethics protocol, with approval from the Animal Research Ethics Board of Chosun University (approval No. CIACUC 2015-A0034).

\section{Patient consent}

The patients provided written informed consent for the pub- lication and the use of their images.

\section{References}

1. McGuire A, Brown JA, Malone C, McLaughlin R, Kerin MJ. Effects of age on the detection and management of breast cancer. Cancers (Basel) 2015;7:908-29.

2. Mangla B, Kohli K. Combination of natural agent with synthetic drug for the breast cancer therapy. Int I Drug Dev Res 2018;10:22-6.

3. Agostinis P, Berg K, Cengel KA, Foster TH, Girotti AW, Gollnick SO, et al. Photodynamic therapy of cancer: an update. CA Cancer J Clin 2011;61:250-81.

4. Silva JN, Filipe $P$, Morlière $P$, Mazière JC, Freitas JP, Cirne de Castro JL, et al. Photodynamic therapies: principles and present medical applications. Biomed Mater Eng 2006; 16:S147-54.

5. O'Connor AE, Gallagher WM, Byrne AT. Porphyrin and nonporphyrin photosensitizers in oncology: preclinical and clinical advances in photodynamic therapy. Photochem Photobiol 2009;85:1053-74.

6. Chin WW, Lau WK, Heng PW, Bhuvaneswari R, Olivo M. Fluorescence imaging and phototoxicity effects of new formulation of chlorin e6-polyvinylpyrrolidone. J Photochem Photobiol B 2006;84:103-10.

7. Petrov $P$, Trukhacheva T, Isakov G, Gavryilov M, Turyn V, Kravchenko E. Photolon an agent for photodynamic diagnosis and therapy: non-clinical and clinical experience. Acta Bio-Optica et Informatica Medica 2004;10:6-7.

8. Ge R, Ahn JC, Shin JI, Bahk CW, He P, Chung PS. An in vitro and in vivo study of combination therapy with Photogem $®$-mediated photodynamic therapy and cisplatin on mouse cancer cells (CT-26). Photomed Laser Surg 2011;29:155-60.

9. Lim HS. Development and optimization of a diode laser for photodynamic therapy. Laser Ther 2011;20:195-203.

10. Beyer W. Systems for light application and dosimetry in photodynamic therapy. J Photochem Photobiol B 1996;36:153-6.

11. Kelland $L$. The resurgence of platinum-based cancer chemotherapy. Nat Rev Cancer 2007;7:573-84.

12. Peddi P, Shi R, Nair B, Ampil F, Mills GM, Jafri SH. Cisplatin, cetuximab, and radiation in locally advanced head and neck squamous cell cancer: a retrospective review. 


\section{Obstetrics \& Gynecology Science}

Tae-Gyu Ahn, et al. Cisplatin effect on photodynamic therapy

Clin Med Insights Oncol 2015;9:1-7.

13. Galluzzi L, Senovilla L, Vitale I, Michels J, Martins I, Kepp $O$, et al. Molecular mechanisms of cisplatin resistance. Oncogene 2012;31:1869-83.

14. Hartmann JT, Lipp HP. Toxicity of platinum compounds. Expert Opin Pharmacother 2003;4:889-901.

15. Stathopoulos GP. Cisplatin: process and future. J BUON 2013;18:564-9.

16. Ali S, Khurshid A, Maqsood M, Rafi M, Khan JA, Zaidi SS, et al. Study of low doses cisplatin synergistic effect on photodynamic outcome of aluminum phythalocyanine on soft tissue sarcoma (RD) cell line. Photodiagn Photodyn Ther 2015;12:146-9.

17. Wei XQ, Ma HQ, Liu AH, Zhang YZ. Synergistic anticancer activity of 5-aminolevulinic acid photodynamic therapy in combination with low-dose cisplatin on Hela cells. Asian Pac J Cancer Prev 2013;14:3023-8.

18. Huang da W, Sherman BT, Lempicki RA. Systematic and integrative analysis of large gene lists using DAVID bioinformatics resources. Nat Protoc 2009;4:44-57.

19. Berg K, Moan J. Lysosomes as photochemical targets. Int J Cancer 1994;59:814-22.

20. Chen B, Pogue BW, Luna JM, Hardman RL, Hoopes PJ, Hasan T. Tumor vascular permeabilization by vasculartargeting photosensitization: effects, mechanism, and therapeutic implications. Clin Cancer Res 2006;12:91723.

21. Johnsson A, Olsson C, Nygren O, Nilsson M, Seiving B, Cavallin-Stahl E. Pharmacokinetics and tissue distribution of cisplatin in nude mice: platinum levels and cisplatin-DNA adducts. Cancer Chemother Pharmacol 1995;37:23-31.

22. Chekulayeva LV, Shevchuk IN, Chekulayev VA, Imarinen K. Hydrogen peroxide, superoxide, and hydroxyl radicals are involved in the phototoxic action of hematoporphyrin derivative against tumor cells. J Environ Pathol Toxicol Oncol 2006;25:51-77.

23. Brozovic A, Ambriović-Ristov A, Osmak M. The relation- ship between cisplatin-induced reactive oxygen species, glutathione, and BCL-2 and resistance to cisplatin. Crit Rev Toxicol 2010;40:347-59.

24. Korbelik M. PDT-associated host response and its role in the therapy outcome. Lasers Surg Med 2006;38:500-8.

25. Xu LL, Warren MK, Rose WL, Gong W, Wang JM. Human recombinant monocyte chemotactic protein and other $\mathrm{C}-\mathrm{C}$ chemokines bind and induce directional migration of dendritic cells in vitro. J Leukoc Biol 1996;60:365-71.

26. Opdenakker G, Froyen G, Fiten P, Proost P, Van Damme J. Human monocyte chemotactic protein-3 (MCP-3): molecular cloning of the cDNA and comparison with other chemokines. Biochem Biophys Res Commun 1993;191:535-42.

27. Jia GQ, Gonzalo JA, Lloyd C, Kremer L, Lu L, MartinezA $C$, et al. Distinct expression and function of the novel mouse chemokine monocyte chemotactic protein-5 in lung allergic inflammation. J Exp Med 1996;184:193951.

28. Wolpe SD, Sherry B, Juers D, Davatelis G, Yurt RW, Cerami A. Identification and characterization of macrophage inflammatory protein 2. Proc Natl Acad Sci U S A 1989;86:612-6.

29. Persson T, Monsef N, Andersson P, Bjartell A, Malm J, Calafat J, et al. Expression of the neutrophil-activating CXC chemokine ENA-78/CXCL5 by human eosinophils. Clin Exp Allergy 2003;33:531-7.

30. Luster AD, Unkeless JC, Ravetch JV. Gamma-interferon transcriptionally regulates an early-response gene containing homology to platelet proteins. Nature 1985;315:672-6.

31. Anestakis D, Petanidis S, Kalyvas S, Nday CM, Tsave O, Kioseoglou E, et al. Mechanisms and applications of interleukins in cancer immunotherapy. Int J Mol Sci 2015;16:1691-710.

32. Pankov R, Yamada KM. Fibronectin at a glance. J Cell Sci 2002;115:3861-3. 